\title{
Upper Bounds for the Distance between a Controllable Switched Linear System and the Set of Uncontrollable Ones
}

\author{
Josep Clotet and M. Dolors Magret \\ Escola Tècnica Superior d'Enginyeria Industrial Barcelona, Universitat Politècnica de Catalunya, \\ Diagonal 647, 08028 Barcelona, Spain \\ Correspondence should be addressed to Josep Clotet; josep.clotet@upc.edu
}

Received 27 February 2013; Revised 2 May 2013; Accepted 7 May 2013

Academic Editor: Xiaojie Su

Copyright (c) 2013 J. Clotet and M. D. Magret. This is an open access article distributed under the Creative Commons Attribution License, which permits unrestricted use, distribution, and reproduction in any medium, provided the original work is properly cited.

\begin{abstract}
The set of controllable switched linear systems is an open and dense set in the space of all switched linear systems. Therefore it makes sense to compute the distance from a controllable system to the nearest uncontrollable one. In the case of a standard system, $\dot{x}(t)=A x(t)+B u(t)$, R. Eising, D. Boley, and W. S. Lu obtain some results for this distance, both in the complex and real cases. In this work we explore this distance, for switched linear systems in the real case, obtaining upper bounds for it. The main contribution of the paper is to prove that a natural generalization of the upper bound obtained by D. Boley and W. S. Lu is true in the case of switched linear systems.
\end{abstract}

\section{Introduction}

A natural question in system theory (and in many other areas of applied mathematics) is whether a certain qualitative property of a system is, or not, preserved under small changes in its parameters (external perturbations, uncertainty on their measure, etc.). If the answer is affirmative, a second natural question concerns the "smallness" of these admissible changes. See, for example, [1, 2].

In particular, the controllability of a system is a generic property; that is to say, the set of controllable systems is open and dense in the corresponding space of matrices. Hence, it makes sense to ask how far a controllable system is from the nearest uncontrollable ones (see, e.g., [2-7]) and to find bounds for the distance between a controllable system and the set of uncontrollable ones (see, e.g., $[2,3,8]$ ).

It is known (Eising [4]) that the problem of computing the distance from a given controllable standard system

$$
\dot{x}(t)=A x(t)+B u(t)
$$

with $A \in M_{n \times n}(\mathbb{C})$ and $B \in M_{n \times p}(\mathbb{C})$ to the set of uncontrollable ones, denoted by $\mu_{\mathbb{C}}(A, B)$, is equivalent to computing the minimum of the smallest singular value of $\left(A-\lambda I_{n} \mid B\right)$ over $\lambda \in \mathbb{C}$ :

$$
\mu_{\mathbb{C}}(A, B)=\min _{\lambda \in \mathbb{C}} \sigma_{n}\left(A-\lambda I_{n} \mid B\right),
$$

this means that there aren't uncontrollable systems in a ball centered in $(A, B)$ with radius $\mu_{\mathbb{C}}(A, B)$, with respect to a given matrix norm.

In this paper we tackle a generalization of this result. As we will see in an example in Section 4, in the case where switched linear systems are considered, the natural generalization does not provide the distance from a controllable switched linear system to the set of uncontrollable ones.

We can also consider, given a controllable standard system

$$
\dot{x}(t)=A x(t)+B u(t)
$$

with $A \in M_{n \times n}(\mathbb{R})$ and $B \in M_{n \times p}(\mathbb{R})$, the distance from this system to the set of uncontrollable (real) systems and denote it by $\mu_{\mathbb{R}}(A, B)$. Actually, complex perturbations of real systems do not always correspond to a system with physical meaning. Note that in this case (Boley and $\mathrm{Lu} \mathrm{[3]}$ )

$$
\mu_{\mathbb{R}}(A, B) \leq \min _{\lambda \in \mathbb{R}} \sigma_{n}\left(A-\lambda I_{n} \mid B\right)
$$


and the equality is not true in general (see the example in [2, page 557]). Obviously $\mu_{\mathbb{C}}(A, B) \leq \mu_{\mathbb{R}}(A, B)$. In particular, any upper bound for $\mu_{\mathbb{R}}(A, B)$ is also an upper bound for $\mu_{\mathbb{C}}(A, B)$.

Many practical systems can be modelled as switched linear systems. Due to their theoretical and practical importance switched linear systems have attracted a lot of interest during the last years. For continuous-time linear switched systems, with time-varying delay, the weighted $\mathscr{H}_{\infty}$ model reduction problem has been investigated in [9] and for continuous-time switched stochastic systems, the problems of dissipativity analysis and dissipativity-based sliding mode control have been studied in [10]. In [11] problems of stability and stabilization for discrete-time switched nonlinear systems with time-varying delay are studied (see also [12] for the study of stability and stabilization problems for a class of discrete-time Takagi-Sugeno fuzzy systems with time-varying state delay). In [13] the position control for a two-wheeled mobile robot is studied and in [14] an application to a dynamic output feedback controller design is described.

Several authors have recently studied controllability and observability of switched linear systems. Algebraic characterization of controllability in [15] shows that controllability is also in this case a generic property. Therefore, the analogous questions arise and it makes sense to study the bounds for the distances from a controllable switched linear system to the nearest uncontrollable one, as it was studied for standard systems. Up to now, no bound for switched linear systems had been obtained, except that in [16] (by the same authors). In this work, we concretely obtain an upper bound for this distance which improves substantially that obtained in [16].

The structure of the paper is as follows.

In Section 2, we summarize the definitions and some properties of norm matrices.

In Section 3, we lay the foundations of the problem to be solved.

In Section 4, we obtain (Theorem 9) an explicit upper bound for the distance between a controllable switched linear system and the set of uncontrollable ones.

Finally, in Section 5, we consider different examples.

\section{Preliminaries}

Let us consider the vector space of $m \times n$ matrices with coefficients in a field $k(k=\mathbb{R}$ or $\mathbb{C})$.

A matrix norm \|\| is a mapping associating to each matrix $M$ a nonnegative number $\|M\|$ having the following properties.

(1) For all matrix $M,\|M\| \geq 0$ and $\|M\|=0$ if, and only if, $M=0$.

(2) For all matrix $M$ and $\lambda \in k,\|\lambda M\|=|\lambda|\|M\|$.

(3) For all matrices $M_{1}$ and $M_{2},\left\|M_{1}+M_{2}\right\| \leq\left\|M_{1}\right\|+$ $\left\|M_{2}\right\|$.

The most frequently used matrix norms are the Frobenius norm and the $p$-norms.
The Frobenius norm of a matrix $M=\left(m_{j}^{i}\right)_{\substack{1 \leq i \leq m \\ 1 \leq j \leq n}}$ is defined as

$$
\|M\|_{F}=\sqrt{\sum_{1 \leq i \leq m} \sum_{1 \leq j \leq n}\left|m_{j}^{i}\right|^{2}}
$$

The matrix $p$-norm is defined for a real number $1 \leq p \leq$ $\infty$ as

$$
\|M\|_{p}=\sup _{x \neq 0} \frac{\|M x\|_{p}}{\|x\|_{p}}=\max _{\|x\|_{p}=1}\|M x\|_{p}
$$

where

$$
\|x\|_{p}=\left(\left|x_{1}\right|^{p}+\cdots+\left|x_{n}\right|^{p}\right)^{1 / p}, \quad 1 \leq p<\infty
$$

and $\|x\|_{\infty}=\max _{1 \leq i \leq n}\left|x_{i}\right|$.

In particular, $\|M\|_{1}=\max _{1 \leq j \leq n} \sum_{i=1}^{m}\left|m_{j}^{i}\right|$. The spectral norm \|\|$_{2}$ is the square root of the maximum eigenvalue of $M^{t} M$ in the real case and $M^{H} M$ in the complex case, where $M^{t}, M^{H}$ denote the transpose and the conjugate transpose of the matrix $M$, respectively. And $\|M\|_{\infty}=$ $\max _{1 \leq i \leq m} \sum_{1 \leq j \leq n}\left|m_{j}^{i}\right|$.

The Frobenius and the $p$-norms satisfy the following inequality (submultiplicative property): for all $m \times n$ matrix $M_{1}$ and $n \times p$ matrix $M_{2},\left\|M_{1} M_{2}\right\| \leq\left\|M_{1}\right\|\left\|M_{2}\right\|$, and some further inequalities, relating them and which are commonly used in matrix analysis,

(i) $\|M\|_{2} \leq\|M\|_{F} \leq \sqrt{n}\|M\|_{2}$,

(ii) $\max _{i, j}\left|m_{j}^{i}\right| \leq\|M\|_{2} \leq \sqrt{m n} \max _{i, j}\left|m_{j}^{i}\right|$,

(iii) $(1 / \sqrt{m})\|M\|_{1} \leq\|M\|_{2} \leq \sqrt{n}\|M\|_{1}$,

(iv) $(1 / \sqrt{n})\|M\|_{\infty} \leq\|M\|_{2} \leq \sqrt{m}\|M\|_{\infty}$.

\section{Set-Up}

Firstly, we will make precise the systems to which the results in Section 4 can be applied. Let us consider a switched linear system $\Sigma$ defined by

$$
\dot{x}(t)=A_{\theta(t)} x(t)+B_{\theta(t)} u(t),
$$

where $A_{\theta(t)} \in M_{n \times n}(\mathbb{R}), B_{\theta(t)} \in M_{n \times p}(\mathbb{R}), x(t) \in \mathbb{R}^{n}$ is the state; $u(t) \in \mathbb{R}^{p}$ is the piecewise continuous input function; and $\theta$ is the piecewise constant signal having range an index set $\Lambda=\{1, \ldots, \ell\}$.

A switching signal (see [15]) is said to be a switching path if it is a function of time. Given an initial time $t_{0}$, a switching path is a function of time.

$\theta:\left[t_{0}, T\right) \rightarrow \Lambda, t_{0}<T \leq \infty$. A switching path $\theta$ is said to be well defined on $\left[t_{0}, T\right)$ if it is defined in $\left[t_{0}, T\right)$ and for all $t \in\left[t_{0}, T\right)$, both $\lim _{s \rightarrow t^{+}} \theta(s)$ and $\lim _{s \rightarrow t^{-}} \theta(s)$ exist and the set

$$
\left\{t \in\left[t_{0}, T\right) \mid \lim _{s \rightarrow t^{+}} \theta(s) \neq \lim _{s \rightarrow t^{-}} \theta(s)\right\}
$$

is finite for any finite sub interval of $\left[t_{0}, T\right.$ ) (in the case where $t=t_{0}$, we will consider $\left.\lim _{s \rightarrow t_{0}^{-}} \theta(s)=\theta\left(t_{0}\right)\right)$. 
Sun-Ge proved in [15] that the system above is controllable if, and only if, $\mathbb{R}^{n}$ is equal to the vector space

$$
\mathscr{V}=\sum_{i_{0}, \ldots, i_{n-1} \in\{1, \ldots, \ell\}}^{j_{1}, \ldots, j_{n-1} \in\{0, \ldots, n-1\}} \operatorname{Im}\left(A_{i_{n-1}}^{j_{n-1}} \cdots A_{i_{1}}^{j_{1}} B_{i_{0}}\right)
$$

where $\operatorname{Im}(M)$ stands for image of matrix $M$. Hence controllability depends only on the set of matrices $\left\{\left(A_{i}, B_{i}\right)_{i \in\{1, \ldots, \ell\}}\right\}$ defining the subsystems of $\Sigma$.

Therefore, the set of matrices $\left\{\left(A_{i}, B_{i}\right)_{i \in\{1, \ldots, \ell\}}\right\}$ defining subsystems of a controllable system is an open dense subset of $M_{n \times n}(\mathbb{R})^{\ell} \times M_{n \times p}(\mathbb{R})^{\ell}$. In particular, for each set of matrices $\left\{\left(A_{i}, B_{i}\right)_{i \in\{1, \ldots, \ell\}}\right\}$ there exists an open neighborhood of this set in $M_{n \times n}(\mathbb{R})^{\ell} \times M_{n \times p}(\mathbb{R})^{\ell}$ with the whole set of matrices in it defining subsystems of a controllable system.

The norm (Frobenius norm, 2-norm, etc.) of the set of matrices $\left\{\left(A_{i}, B_{i}\right)_{i \in\{1, \ldots, \ell\}}\right\}$ is taken as

$$
\left\|\left\{\left(A_{i}, B_{i}\right)_{i \in\{1, \ldots, \ell\}}\right\}\right\|=\left\|\left(A_{1}\left|B_{1}\right| \cdots\left|A_{\ell}\right| B_{\ell}\right)\right\|
$$

and thus the distance between two sets of matrices is

$$
\begin{gathered}
d\left(\left\{\left(A_{i}, B_{i}\right)_{i \in\{1, \ldots, \ell\}}\right\},\left\{\left(A_{i}^{\prime}, B_{i}^{\prime}\right)_{i \in\{1, \ldots, \ell\}}\right\}\right) \\
=\left\|\left\{\left(A_{i}^{\prime}-A_{i}, B_{i}^{\prime}-B_{i}\right)_{i \in\{1, \ldots, \ell\}}\right\}\right\| .
\end{gathered}
$$

Given a controllable system $\Sigma$ with subsystems defined by the set of matrices $\left\{\left(A_{i}, B_{i}\right)_{i \in\{1, \ldots, \ell\}}\right\}$, the distance (with regard to a given matrix norm) from this system to the nearest uncontrollable one is

$$
\begin{aligned}
\mu_{\mathbb{R}} & \left(\left\{\left(A_{i}, B_{i}\right)_{i \in\{1, \ldots, \ell\}}\right\}\right) \\
& =\min _{\delta A_{i} \in M_{n \times n}(\mathbb{R}), \delta B_{i} \in M_{n \times p}(\mathbb{R})}\left\|\left\{\left(\delta A_{i}, \delta B_{i}\right)_{i \in\{1, \ldots, \ell\}}\right\}\right\|
\end{aligned}
$$

with $\left\{\left(A_{i}+\delta A_{i}, B_{i}+\delta B_{i}\right)_{i \in\{1, \ldots, \ell\}}\right\}$ being the set of matrices defining the subsystems of an uncontrollable system.

\section{Upper Bound for the Distance}

Note that if we generalize, in a natural way, (2) to the case of switched linear systems and define

$$
\begin{aligned}
\mu_{\mathbb{C}} & \left(\left\{\left(A_{i}, B_{i}\right)_{i \in\{1, \ldots, \ell\}}\right\}\right) \\
& =\min _{\delta A_{i} \in M_{n \times n}(\mathbb{C}), \delta B_{i} \in M_{n \times p}(\mathbb{C})}\left\|\left\{\left(\delta A_{i}, \delta B_{i}\right)_{i \in\{1, \ldots, \ell\}}\right\}\right\|
\end{aligned}
$$

with $\left\{\left(A_{i}+\delta A_{i}, B_{i}+\delta B_{i}\right)_{i \in\{1, \ldots, \ell\}}\right\}$ being the set of matrices defining the subsystems of an uncontrollable system, then this distance $\mu_{\mathbb{C}}\left(\left\{\left(A_{i}, B_{i}\right)_{i \in\{1, \ldots, \ell\}}\right\}\right)$ is not equal to

$$
\min _{\lambda_{1}, \ldots, \lambda_{\ell} \in \mathbb{C}} \sigma_{n}\left(A_{1}-\lambda_{1} I_{n}\left|B_{1}\right| \cdots\left|A_{\ell}-\lambda_{\ell} I_{n}\right| B_{\ell}\right)
$$

as the following example shows.
Example 1. Consider a switched linear system with two subsystems defined by matrices

$$
\begin{gathered}
A_{1}=\left(\begin{array}{ccc}
0 & 0 & 0 \\
0 & 2 & 0 \\
0.1 & 1 & 2
\end{array}\right), \quad A_{2}=\left(\begin{array}{lll}
0 & 1 & 1 \\
0 & 0 & 1 \\
0 & 0 & 1
\end{array}\right), \\
B_{1}=B_{2}=\left(\begin{array}{l}
1 \\
0 \\
0
\end{array}\right) .
\end{gathered}
$$

This system is controllable, as it is straightforward to be checked $(\operatorname{dim} \mathscr{V}=3)$ and

$$
\begin{aligned}
& \min _{\lambda_{1}, \lambda_{2} \in \mathbb{C}} \sigma_{3}\left(A_{1}-\lambda_{1} I_{3}\left|B_{1}\right| \cdots\left|A_{2}-\lambda_{2} I_{3}\right| B_{2}\right) \\
& =\min _{\lambda_{1}, \lambda_{2} \in \mathbb{C}} \sigma_{3}\left(\begin{array}{cccccccc}
\lambda_{1} & 0 & 0 & 1 & -\lambda_{2} & 1 & 1 & 1 \\
0 & 2-\lambda_{1} & 0 & 0 & 0 & -\lambda_{2} & 1 & 0 \\
0.1 & 1 & 2-\lambda_{1} & 0 & 0 & 0 & 1-\lambda_{2} & 0
\end{array}\right) \\
& =0.348160,
\end{aligned}
$$

but the system consisting of two subsystems defined by

$$
\begin{gathered}
A_{1}=\left(\begin{array}{lll}
0 & 0 & 0 \\
0 & 2 & 0 \\
0 & 1 & 2
\end{array}\right), \quad A_{2}=\left(\begin{array}{lll}
0 & 1 & 1 \\
0 & 0 & 1 \\
0 & 0 & 1
\end{array}\right), \\
B_{1}=B_{2}=\left(\begin{array}{l}
1 \\
0 \\
0
\end{array}\right),
\end{gathered}
$$

is uncontrollable and the distance (both considering the Frobenius norm and the 2-norm) between this system and the previous one is $0.1<0.348160$.

The main result in this section is the generalization of inequality (4) to the case of switched linear systems. The natural generalization seems to be

$$
\begin{aligned}
\mu_{\mathbb{R}}( & \left.\left\{\left(A_{i}, B_{i}\right)_{i \in\{1, \ldots, \ell\}}\right\}\right) \\
& \leq \min _{\lambda_{1}, \ldots, \lambda_{\ell} \in \mathbb{R}} \sigma_{n}\left(A_{1}-\lambda_{1} I_{n}\left|B_{1}\right| \cdots\left|A_{\ell}-\lambda_{\ell} I_{n}\right| B_{\ell}\right) .
\end{aligned}
$$

This generalization is proved in Theorem 9.

Consider the switched linear system (8) and let us assume that this system is controllable. For each $i \in\{1, \ldots, \ell\}$, let us consider the vector subspace

$$
\sum_{\substack{k_{1}, \ldots, k_{\ell} \in\{0,1\} \\ k_{1}+\cdots+k_{\ell}=1 \\ j_{1}, \ldots, j_{n-2} \in\{0,1, \ldots, n-2\} \\ j_{1} \cdots+j_{n-2} \leq n-2 \\ h_{1}, \ldots, h_{n-2} \in\{1, \ldots, \ell\} \\ A_{i} \neq A_{h_{1}}}}\left[\sum_{0 \leq j \leq n-1} \operatorname{Im}\left(A_{i}^{j}\left(A_{h_{1}}^{j_{1}} \cdots A_{h_{n-2}}^{j_{n-2}} B_{1}^{k_{1}} \cdots B_{\ell}^{k_{\ell}}\right)\right)\right]
$$

and let us denote by $G_{i}(\Sigma)$ the matrix having as columns the generators of the vector subspace above and by $G(\Sigma)$ the matrix

$$
G(\Sigma)=\left(G_{1}(\Sigma)|\cdots| G_{\ell}(\Sigma)\right) .
$$

Throughout the paper, the notation $\operatorname{rk} M$ will denote the rank of matrix $M$. 
Lemma 2. The rank of matrix $G(\Sigma)$ (written $\mathrm{rk} G(\Sigma)$ ) is equal to the dimension of the vector space

$$
\sum_{\substack{k_{1}, \ldots, k_{\ell} \in\{0,1\} \\ k_{1}+\cdots+k_{\ell}=1 \\ 1, \ldots, j_{n-1} \in\{0,1, \ldots, n-1\} \\ i_{1}, \ldots, i_{n-1} \in\{1, \ldots, \ell\}}} \operatorname{Im}\left(A_{i_{1}}^{j_{1}} \cdots A_{i_{n-1}}^{j_{n-1}} B_{1}^{k_{1}} \cdots B_{\ell}^{k_{\ell}}\right) .
$$

Proof. It is a straightforward consequence of CayleyHamilton Theorem and the fact that this vector subspace and $\mathscr{V}$ defined above are the same subspace.

As a consequence of Lemma 2, another characterization of controllable switched linear systems is that system $\Sigma$ is controllable if, and only if, $\operatorname{rk} G(\Sigma)=n$.

A first upper bound for the distance, from our system to the set of uncontrollable ones, may be deduced from the singular value decomposition of matrix $G(\Sigma)$, in a similar way to the one in [3], in the case where $\operatorname{rk} G_{i}(\Sigma)=n$, for all $i \in\{1, \ldots, \ell\}$. Namely, let $P, Q$ be orthogonal matrices such that

$$
G(\Sigma)=P^{t}\left(\operatorname{diag}\left(\sigma_{1}, \ldots, \sigma_{n}\right) \mid 0\right) Q
$$

where $\sigma_{1} \geq \cdots \geq \sigma_{n}$ are the singular values of $G(\Sigma)$. Then the following statement holds.

Theorem 3 (see [16]). Given a controllable switched linear system $\Sigma$ consisting of subsystems defined by a set of matrices $\left\{\left(A_{i}, B_{i}\right)_{i \in\{1, \ldots, \ell\}}\right\}$, such that $\operatorname{rk} G_{i}(\Sigma)=n$, for all $i \in\{1, \ldots, \ell\}$, with regard to the 2-norm,

$$
\mu_{\mathbb{R}}\left(\left\{\left(A_{i}, B_{i}\right)_{i \in\{1, \ldots, \ell\}}\right\}\right) \leq \sum_{i \in\{1, \ldots, \ell\}} \sigma_{n}\left(1+\frac{\left\|A_{i}^{C}\right\|_{2}}{\sigma_{n, i}}\right),
$$

where $A_{i}^{C}$ is the companion matrix for $A_{i}$ and $\sigma_{1, i} \geq \cdots \geq \sigma_{n, i}$ are the singular values of $G_{i}(\Sigma)$.

Remember that, given a square matrix $A$ with characteristic polynomial $Q(t)=t^{n}+a_{1} t^{n-1}+\cdots+a_{n-1} t+a_{n}$, the matrix

$$
\left(\begin{array}{ccccc}
0 & 0 & \cdots & 0 & -a_{n} \\
1 & 0 & \cdots & 0 & -a_{n-1} \\
0 & 1 & \cdots & 0 & -a_{n-2} \\
\cdots & \cdots & & \cdots & \cdots \\
0 & 0 & \cdots & 1 & -a_{1}
\end{array}\right)
$$

is called the companion matrix of matrix $A$.

Proof. First of all, note that $\left\{\left(A_{i}, B_{i}\right)_{i \in\{1, \ldots, \ell\}}\right\}$ are associated to a controllable system if, and only if, $\left\{\left(P A_{i} P^{t}, P B_{i}\right)_{i \in\{1, \ldots, \ell\}}\right\}$ are also associated to a controllable system.

Denoting by $\mathscr{P}$

$$
\operatorname{diag}\left(\left(\begin{array}{cc}
P^{t} & \\
& I_{p}
\end{array}\right), \ldots,\left(\begin{array}{ll}
P^{t} & \\
& I_{p}
\end{array}\right)\right)
$$

and since

$$
\begin{aligned}
& P\left(A_{1}-X_{1}\left|B_{1}-Y_{1}\right| \cdots\left|A_{\ell}-X_{\ell}\right| B_{\ell}-Y_{\ell}\right) \mathscr{P} \\
& =\left(P\left(A_{1}-X_{1}\right) P^{t}\left|P\left(B_{1}-Y_{1}\right)\right| \cdots\left|P\left(A_{\ell}-X_{\ell}\right) P^{t}\right|\right. \\
& \left.P\left(B_{\ell}-Y_{\ell}\right)\right)
\end{aligned}
$$

and $P$ and $\mathscr{P}$ are orthogonal matrices, we deduce that

$$
\begin{aligned}
& \left\|\left(A_{1}-X_{1}\left|B_{1}-Y_{1}\right| \cdots\left|A_{\ell}-X_{\ell}\right| B_{\ell}-Y_{\ell}\right)\right\|_{2} \\
& =\|\left(P\left(A_{1}-X_{1}\right) P^{t}\left|P\left(B_{1}-Y_{1}\right)\right| \cdots\left|P\left(A_{\ell}-X_{\ell}\right) P^{t}\right|\right. \\
& \left.\quad P\left(B_{\ell}-Y_{\ell}\right)\right) \|_{2} .
\end{aligned}
$$

Therefore

$$
\mu_{\mathbb{R}}\left(\left\{\left(A_{i}, B_{i}\right)_{i \in\{1, \ldots, \ell\}}\right\}\right)=\mu_{\mathbb{R}}\left(\left\{\left(P A_{i} P^{t}, P B_{i}\right)_{i \in\{1, \ldots, \ell\}}\right\}\right) .
$$

Let us consider $P B_{i}=\left(\begin{array}{c}B_{i}^{1} \\ B_{i}^{2}\end{array}\right)$, with $B_{i}^{1} \in M_{(n-1) \times p}(\mathbb{R})$, for $i \in\{1, \ldots, \ell\}$.

We can write

$$
\begin{aligned}
B_{i}^{2} & \left.=e_{n}^{t}\left(P B_{i}\right)=e_{n}^{t}\left(P G(\Sigma)\left(0 \cdots 0 \stackrel{\left(j_{i}\right)}{I_{p}} 0 \cdots 0\right)^{t}\right)\right) \\
& =e_{n}^{t}\left(\left(\operatorname{diag}\left(\sigma_{1}, \ldots, \sigma_{n}\right) \mid 0\right) Q\left(\begin{array}{ccc}
0 & \cdots & \left(j_{i}\right) \\
I_{p} & 0 \cdots 0
\end{array}\right)^{t}\right),
\end{aligned}
$$

where $j_{i}$ indicates the column $j_{i}$ of matrix $G(\Sigma)$ where matrix $B_{i}$ first appears, with $e_{1}, \ldots, e_{n}$ being the natural basis of the Euclidean space $\mathbb{R}^{n}$. Then

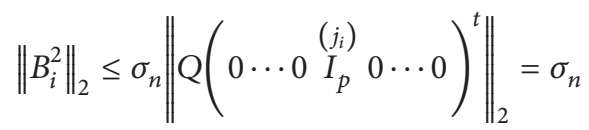

since $Q$ is an orthogonal matrix.

It is straightforward to check that $A_{i} G_{i}(\Sigma)=G_{i}(\Sigma)(I \otimes$ $A_{i}^{C}$ ), where $I$ is the identity matrix of suitable order. Then if we denote by $G_{i}(\Sigma)^{\dagger}$ the Moore-Penrose inverse of matrix $G_{i}(\Sigma)$, $A_{i}=G_{i}(\Sigma)\left(I \otimes A_{i}^{C}\right) G_{i}(\Sigma)^{\dagger}$ and

$$
\begin{aligned}
P A_{i} P^{t}= & P G_{i}(\Sigma)\left(I \otimes A_{i}^{C}\right) G_{i}(\Sigma)^{\dagger} P^{t} \\
= & P\left(G(\Sigma)\left(0 \cdots 0{\stackrel{(i)}{I_{c_{i}}}}^{t} \cdots 0\right)^{t}\right)\left(I \otimes A_{i}^{C}\right) G_{i}(\Sigma)^{\dagger} P^{t} \\
= & \left(\operatorname{diag}\left(\sigma_{1}, \ldots, \sigma_{n}\right) \mid 0\right) Q\left(0 \cdots 0 \stackrel{(i)}{I}_{c_{i}} 0 \cdots 0\right)^{t} \\
& \times\left(I \otimes A_{i}^{C}\right) G_{i}(\Sigma)^{\dagger} P^{t},
\end{aligned}
$$

where $c_{i}$ is the number of columns of matrix $G_{i}(\Sigma)$. 
In an analogous way, let us consider

$$
P A_{i} P^{t}=\left(\begin{array}{rr}
A_{i}^{1} & A_{i}^{2} \\
A_{i}^{3} & A_{i}^{4}
\end{array}\right)
$$

with $A_{i}^{1} \in M_{(n-1) \times(n-1)}(\mathbb{R})$,

$$
\begin{aligned}
& A_{i}^{3}=e_{n}^{t}\left(\operatorname{diag}\left(\sigma_{1}, \ldots, \sigma_{n}\right) \mid 0\right) Q \\
& \times\left(0 \cdots 0 \stackrel{(i)}{I_{c_{i}}} 0 \cdots 0\right)^{t}\left(I \otimes A_{i}^{C}\right) Q_{i}^{t} \\
& \times\left(\operatorname{diag}\left(\frac{1}{\sigma_{1, i}}, \ldots, \frac{1}{\sigma_{n, i}}\right)\right) P_{i} P^{t}\left(\begin{array}{c}
I_{n-1} \\
0
\end{array}\right),
\end{aligned}
$$

where $\sigma_{1, i} \geq \cdots \geq \sigma_{n, i}$, are the singular values of $G_{i}(\Sigma)$ $\left(G_{i}(\Sigma)=P_{i}^{t}\left(\operatorname{diag}\left(\sigma_{1, i}, \ldots, \sigma_{n, i}\right) \mid 0\right) Q_{i}\right)$. Therefore

$$
\left\|A_{i}^{3}\right\|_{2} \leq \sigma_{n}\left\|\left(I \otimes A_{i}^{C}\right)\right\|_{2}\left\|G_{i}(\Sigma)^{\dagger}\right\|_{2} \leq \frac{\sigma_{n}}{\sigma_{n, i}}\left\|A_{i}^{C}\right\|_{2} .
$$

Let us set the blocks $B_{i}^{2}, A_{i}^{3}$ to zero, for all $i$; it is obvious that the system thus obtained is uncontrollable. The real perturbation we have committed has a norm which is bounded by $\sigma_{n}+\left(\left\|A_{i}^{C}\right\|_{2} \sigma_{n} / \sigma_{(n, i)}\right)$, for all $i \in\{1, \ldots, \ell\}$, and the statement follows.

Observe that, in the case where $\ell=1$ (there is only one subsystem) we obtain the same result as that in [3] because the product $P_{i} P^{t}$ in (34) is equal to $I_{n}$, and therefore $\left\|A_{i}^{3}\right\|_{2} \leq$ $\left(\sigma_{n} / \sigma_{n-1}\right)\left\|A_{i}^{C}\right\|_{2}$.

In order to obtain an improved upper bound, we need the following matrix.

Let us consider a switched linear system with subsystems defined by the set of matrices $\left\{\left(A_{i}, B_{i}\right)_{i \in\{1, \ldots, \ell\}}\right\}$. Given $\lambda_{1}, \ldots, \lambda_{\ell} \in \mathbb{R}$, we will denote by $M\left(\Sigma, \lambda_{1}, \ldots, \lambda_{\ell}\right)$ the following matrix:

$$
M\left(\Sigma, \lambda_{1}, \ldots, \lambda_{\ell}\right)=\left(A_{1}-\lambda_{1} I_{n}\left|B_{1}\right| \cdots\left|A_{\ell}-\lambda_{\ell} I_{n}\right| B_{\ell}\right) .
$$

Lemma 4. Given a switched linear system $\sum$ with subsystems defined by a set of matrices $\left\{\left(A_{i}, B_{i}\right)_{i \in\{1, \ldots, \ell\}}\right\}$,

$$
\operatorname{rk} G(\Sigma) \leq \operatorname{rk} M\left(\Sigma, \lambda_{1}, \ldots, \lambda_{\ell}\right), \quad \forall\left(\lambda_{1}, \ldots, \lambda_{\ell}\right) \in \mathbb{R}^{\ell} .
$$

Proof. Let us assume that $\lambda_{1}^{0}, \ldots, \lambda_{\ell}^{0} \in \mathbb{R}$ are such that

$$
\min _{\lambda_{1}, \ldots, \lambda_{\ell} \in \mathbb{R}} \operatorname{rk} M\left(\Sigma, \lambda_{1}, \ldots, \lambda_{\ell}\right)=\operatorname{rk} M\left(\Sigma, \lambda_{1}^{0}, \ldots, \lambda_{\ell}^{0}\right)
$$

Note that in the case where $\operatorname{rk} M\left(\Sigma, \lambda_{1}^{0}, \ldots, \lambda_{\ell}^{0}\right)=n$, the statement is obvious. Let us assume that the rank of $M\left(\Sigma, \lambda_{1}^{0}, \ldots, \lambda_{\ell}^{0}\right)=n-m, m>0$. Then there exist linearly independent vectors $v_{1}, \ldots, v_{m} \in \mathbb{R}^{n}$ such that

$$
v_{1}^{t} M\left(\Sigma, \lambda_{1}^{0}, \ldots, \lambda_{\ell}^{0}\right)=\cdots=v_{m}^{t} M\left(\Sigma, \lambda_{1}^{0}, \ldots, \lambda_{\ell}^{0}\right)=0 .
$$

Therefore

$$
\begin{gathered}
v_{i}^{t}\left(A_{1}-\lambda_{1} I_{n}\right)=\cdots=v_{i}^{t}\left(A_{\ell}-\lambda_{\ell} I_{n}\right)=0, \quad i \in\{1, \ldots, m\}, \\
v_{i}^{t} B_{1}=\cdots=v_{i}^{t} B_{\ell}=0, \quad i \in\{1, \ldots, m\} .
\end{gathered}
$$

It is straightforward to check that $v_{1}, \ldots, v_{m}$ are left eigenvectors of matrices $A_{1}, \ldots, A_{\ell}$ and therefore

$$
v_{1}^{t} G(\Sigma)=\cdots=v_{m}^{t} G(\Sigma)=0 .
$$

Thus $\operatorname{rk} G(\Sigma) \leq n-m$.

As a consequence of Lemma 4, we can conclude that if there exist $\lambda_{1}^{0}, \ldots, \lambda_{\ell}^{0} \in \mathbb{R}$ such that $\operatorname{rk} M\left(\Sigma, \lambda_{1}^{0}, \ldots, \lambda_{\ell}^{0}\right)<n$, the system can not be controllable.

Remark 5. Note that there exist uncontrollable systems such that for all $\lambda_{1}, \ldots, \lambda_{\ell} \in \mathbb{R}, \operatorname{rk} M\left(\Sigma, \lambda_{1}, \ldots, \lambda_{\ell}\right)=n$. For example, we consider a system $\Sigma$ with subsystems defined by matrices

$$
\begin{gathered}
A_{1}=\left(\begin{array}{lll}
0 & 0 & 0 \\
0 & 2 & 0 \\
0 & 1 & 2
\end{array}\right), \quad A_{2}=\left(\begin{array}{lll}
0 & 1 & 1 \\
0 & 0 & 1 \\
0 & 0 & 1
\end{array}\right), \\
B_{1}=B_{2}=\left(\begin{array}{l}
1 \\
0 \\
0
\end{array}\right) .
\end{gathered}
$$

It is straightforward that $\operatorname{rk} G(\Sigma)=1$ and that the rank of $M\left(\Sigma, \lambda_{1}, \lambda_{2}\right)=3$, for all $\lambda_{1}, \lambda_{2} \in \mathbb{R}$.

In particular, we may obtain a new upper bound for the distance between a controllable system and the set of uncontrollable ones, with no restriction on $\operatorname{rk} G_{i}(\Sigma), i \in\{1$, $\ldots, \ell\}$.

Theorem 6. Given a controllable system with subsystems defined by the set of matrices $\left\{\left(A_{i}, B_{i}\right)_{i \in\{1, \ldots, \ell\}}\right\}$,

$$
\mu_{\mathbb{R}}\left(\left\{\left(A_{i}, B_{i}\right)_{i \in\{1, \ldots, \ell\}}\right\}\right) \leq d\left(\left\{\left(A_{i}, B_{i}\right)_{i \in\{1, \ldots, \ell\}}\right\}, \mathscr{X}\right),
$$

where $\mathscr{X}=\left\{\left\{\left(A_{i}, B_{i}\right)_{i \in\{1, \ldots, \ell\}}\right\} \mid \exists \lambda_{1}, \ldots, \lambda_{\ell} \in \mathbb{R}\right.$ such that $\left.\operatorname{rk} M\left(\sum, \lambda_{1}, \ldots, \lambda_{\ell}\right) \leq n-1\right\}$.

Proof. It is easily deduced from Lemma 4.

In the particular case of an uncontrollable system with $\operatorname{rk} G(\Sigma)=n-1$, the results above may be summarized as follows.

Theorem 7. Given an uncontrollable switched linear system with subsystems defined by the set of matrices $\left\{\left(A_{i}, B_{i}\right)_{i \in\{1, \ldots, \ell\}}\right\}$ with $\operatorname{rk} G(\Sigma)=n-1$, there exist $\lambda_{1}^{0}, \ldots, \lambda_{\ell}^{0} \in \mathbb{R}$ such that $\operatorname{rk} M\left(\sum, \lambda_{1}^{0}, \ldots, \lambda_{\ell}^{0}\right)=n-1$.

Proof. Let us consider the controllability canonical form of $\left\{\left(A_{i}, B_{i}\right)_{i \in\{1, \ldots, \ell\}}\right\}$

$$
\left\{\left(\left(\begin{array}{cc}
A_{i}^{1} & A_{i}^{2} \\
0 & A_{i}^{3}
\end{array}\right),\left(\begin{array}{c}
B_{i}^{1} \\
0
\end{array}\right)\right)_{i \in\{1, \ldots, \ell\}}\right\} .
$$


Since $\operatorname{rk} G(\Sigma)=n-1, A_{i}^{3}=\left(a_{i}^{3}\right), 1 \leq i \leq \ell$, it suffices to take $\lambda_{1}^{0}=a_{1}^{3}, \ldots, \lambda_{\ell}^{0}=a_{\ell}^{3}$.

Remark 8. Note that given a controllable system $(\operatorname{rk} G(\Sigma)=$ $n$ ) it is possible that the nearest uncontrollable one is a system $\Sigma^{\prime}$ such that $\operatorname{rk} G\left(\Sigma^{\prime}\right)<n-1$. For example, let us consider the system with subsystems defined by

$$
\begin{aligned}
A_{1}=\left(\begin{array}{lll}
0 & 0 & 0 \\
1 & 0 & 0 \\
0 & 1 & 0
\end{array}\right), & B_{1}=\left(\begin{array}{l}
0 \\
0 \\
1
\end{array}\right), \\
A_{2}=\left(\begin{array}{lll}
0 & 0 & 0 \\
1 & 0 & 0 \\
0 & 1 & 0
\end{array}\right), & B_{2}=\left(\begin{array}{c}
0.1 \\
0 \\
0
\end{array}\right) .
\end{aligned}
$$

It is easy to check that this system is controllable and that the nearest uncontrollable one is a system $\Sigma^{\prime}$ with subsystems defined by

$$
\begin{array}{ll}
A_{1}=\left(\begin{array}{lll}
0 & 0 & 0 \\
1 & 0 & 0 \\
0 & 1 & 0
\end{array}\right), & B_{1}=\left(\begin{array}{l}
0 \\
0 \\
1
\end{array}\right), \\
A_{2}=\left(\begin{array}{lll}
0 & 0 & 0 \\
1 & 0 & 0 \\
0 & 1 & 0
\end{array}\right), & B_{2}=\left(\begin{array}{l}
0 \\
0 \\
0
\end{array}\right),
\end{array}
$$

which is such that $\operatorname{rk} G\left(\Sigma^{\prime}\right)=1$.

More concretely, there is no uncontrollable system with distance (with regard to the Frobenius norm) less than 0.1, from the first system.

Finally, the following upper bound is obtained.

Theorem 9. Given a controllable system $\Sigma$ with subsystems defined by the set of matrices $\left\{\left(A_{i}, B_{i}\right)_{i \in\{1, \ldots, \ell\}}\right\}$, the distance (with regard to the Frobenius norm) from this system to the nearest uncontrollable one is

$$
\mu_{\mathbb{R}}\left(\left\{\left(A_{i}, B_{i}\right)_{i \in\{1, \ldots, \ell\}}\right\}\right) \leq \min _{\lambda_{1}, \ldots, \lambda_{\ell} \in \mathbb{R}} \sigma_{n}\left(M\left(\Sigma, \lambda_{1}, \ldots, \lambda_{\ell}\right)\right)
$$

and the distance (with regard to the 2-norm) from this system to the nearest uncontrollable one is

$$
\mu_{\mathbb{R}}\left(\left\{\left(A_{i}, B_{i}\right)_{i \in\{1, \ldots, \ell\}}\right\}\right) \leq \min _{\lambda_{1}, \ldots, \lambda_{\ell} \in \mathbb{R}} \sigma_{n}\left(M\left(\Sigma, \lambda_{1}, \ldots, \lambda_{\ell}\right)\right),
$$

where $\sigma_{n}\left(M\left(\Sigma, \lambda_{1}, \ldots, \lambda_{\ell}\right)\right)$ is the smallest singular value of the matrix $M\left(\Sigma, \lambda_{1}, \ldots, \lambda_{\ell}\right)$.

Proof. The statement summarizes the results above. We apply theorem in the appendix (Eckart-Young-Mirsky matrix approximation theorem) to the matrix $M\left(\Sigma, \lambda_{1}, \ldots, \lambda_{\ell}\right)$ which has full rank $r=n$ for any values of $\lambda_{1}, \ldots, \lambda_{\ell} \in \mathbb{R}$, because system $\Sigma$ is controllable. A perturbed uncontrollable system $\Sigma^{\prime}$ would have associated the matrix $M\left(\Sigma^{\prime}, \lambda_{1}, \ldots, \lambda_{\ell}\right)$ with rank $k=n-1$. Theorem in the appendix shows that the minimal distance from $M\left(\Sigma, \lambda_{1}, \ldots, \lambda_{\ell}\right)$ to $M\left(\Sigma^{\prime}, \lambda_{1}, \ldots, \lambda_{\ell}\right)$

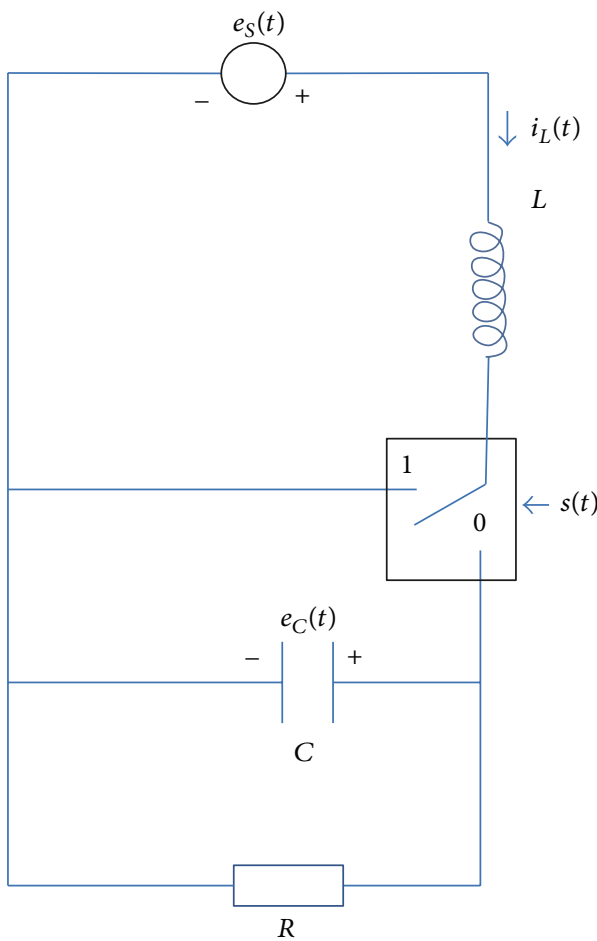

Figure 1: Boost converter.

(with regard to the Frobenius norm or the 2-norm) is equal to the smallest singular value of matrix $M\left(\Sigma, \lambda_{1}, \ldots, \lambda_{\ell}\right)$. The nearest uncontrollable system is obtained minimizing the values of $\lambda_{1}, \ldots, \lambda_{\ell} \in \mathbb{R}$.

\section{Examples}

Example 1. Let us consider a boost converter (see [17]) which is represented in Figure 1 and with the equations

$$
\left(\begin{array}{c}
\dot{e}_{C}(t) \\
\dot{i}_{L}(t)
\end{array}\right)=\left(\begin{array}{cc}
-\frac{1}{R C} & \frac{1}{C} \\
-\frac{1}{L} & 0
\end{array}\right)\left(\begin{array}{l}
e_{C}(t) \\
i_{L}(t)
\end{array}\right)+\left(\begin{array}{c}
0 \\
\frac{e_{S}}{L}
\end{array}\right)
$$

if $s(t)=0$, and

$$
\left(\begin{array}{c}
\dot{e}_{C}(t) \\
\dot{i}_{L}(t)
\end{array}\right)=\left(\begin{array}{cc}
-\frac{1}{R C} & 0 \\
0 & 0
\end{array}\right)\left(\begin{array}{c}
e_{C}(t) \\
i_{L}(t)
\end{array}\right)+\left(\begin{array}{c}
0 \\
\frac{e_{S}}{L}
\end{array}\right)
$$

if $s(t)=1$.

Denoting $t=\tau T$, with $T$ being the switching time period,

$$
\left(\begin{array}{c}
\dot{e}_{C}(\tau) \\
\dot{i}_{L}(\tau)
\end{array}\right)=\left(\begin{array}{cc}
-\frac{T}{R C} & \frac{T}{C} \\
-\frac{T}{L} & 0
\end{array}\right)\left(\begin{array}{l}
e_{C}(\tau) \\
i_{L}(\tau)
\end{array}\right)+\left(\begin{array}{c}
0 \\
\frac{T}{L} e_{S}
\end{array}\right)
$$


if $s(t)=0$, and

$$
\left(\begin{array}{c}
\dot{e}_{C}(\tau) \\
\dot{i}_{L}(\tau)
\end{array}\right)=\left(\begin{array}{cc}
-\frac{T}{R C} & 0 \\
0 & 0
\end{array}\right)\left(\begin{array}{c}
e_{C}(\tau) \\
i_{L}(\tau)
\end{array}\right)+\left(\begin{array}{c}
0 \\
\frac{T}{L} e_{S}
\end{array}\right)
$$

if $s(t)=1$.

Thus the system can be described as a switched linear system $\Sigma\left(R, C, L, T, e_{S}\right)$ :

$$
\begin{aligned}
& \dot{x}(\tau)=A_{1} x(\tau)+B_{1} u(\tau), \\
& \dot{x}(\tau)=A_{2} x(\tau)+B_{2} u(\tau)
\end{aligned}
$$

with $u(\tau)=1$,

$$
\begin{array}{cc}
A_{1}=\left(\begin{array}{cc}
-\frac{T}{R C} & \frac{T}{C} \\
-\frac{T}{L} & 0
\end{array}\right), & B_{1}=\left(\begin{array}{c}
0 \\
\frac{T}{L} e_{S}
\end{array}\right), \\
A_{2}=\left(\begin{array}{cc}
-\frac{T}{R C} & 0 \\
0 & 0
\end{array}\right), & B_{2}=\left(\begin{array}{c}
0 \\
\frac{T}{L} e_{S}
\end{array}\right) .
\end{array}
$$

Assume that $e_{S}(t)=5 \mathrm{~V}, L=0.75 \mathrm{mH}$, the switching time period is $T=20 \mu \mathrm{s}, C=60 \mu \mathrm{F}$, and $R=20 \Omega$. Then the system obtained, which we will denoted by $\Sigma$, has

$$
\begin{array}{ll}
A_{1}=\left(\begin{array}{cc}
-\frac{1}{60} & \frac{1}{3} \\
-\frac{2}{75} & 0
\end{array}\right), & B_{1}=\left(\begin{array}{c}
0 \\
\frac{2}{15}
\end{array}\right), \\
A_{2}=\left(\begin{array}{cc}
-\frac{1}{60} & 0 \\
0 & 0
\end{array}\right), & B_{2}=\left(\begin{array}{c}
0 \\
\frac{2}{15}
\end{array}\right) .
\end{array}
$$

In this case,

$$
\begin{gathered}
G_{1}(\Sigma)=\left(\begin{array}{lllll}
B_{1} & A_{1} B_{1} & B_{2} & A_{1} B_{2}
\end{array}\right)=\left(\begin{array}{cccc}
0 & \frac{2}{45} & 0 & \frac{2}{45} \\
\frac{2}{15} & 0 & \frac{2}{15} & 0
\end{array}\right), \\
G_{2}(\Sigma)=\left(\begin{array}{lllll}
B_{1} & A_{2} B_{1} & B_{2} & A_{2} B_{2}
\end{array}\right)=\left(\begin{array}{cccc}
0 & 0 & 0 & 0 \\
\frac{2}{15} & 0 & \frac{2}{15} & 0
\end{array}\right) .
\end{gathered}
$$

System $\Sigma$ is controllable, since $\operatorname{rk} G(\Sigma)=\operatorname{rk}\left(G_{1}(\Sigma)\right.$ | $\left.G_{2}(\Sigma)\right)=2$. Note that Theorem 3 cannot be applied because $\operatorname{rk} G_{2}(\Sigma)<2$, but applying Theorem 9, an upper bound for $\mu_{\mathbb{R}}\left(\left\{\left(A_{i}, B_{i}\right)_{i \in\{1,2\}}\right\}\right)$ with regard to the 2-norm can be obtained:

$$
\min _{\lambda_{1}, \lambda_{2} \in \mathbb{R}} \sigma_{2}\left(M\left(\Sigma, \lambda_{1}, \lambda_{2}\right)\right)=0.186217
$$

Note that for any real value of $R \neq 0, C \neq 0, L \neq 0, T \neq 0$ and for any constant function $e_{S} \neq 0$, system $\Sigma\left(R, C, L, T, e_{S}\right)$ is controllable. In order to obtain an uncontrollable system we should apply a parameter variation to the values of the parameters, setting $e_{S}=0$, or $T=0$, or $L=\infty$, or $C=$ $\infty$. Straightforward calculations show that the distance, with regard to the 2-norm, from system $\Sigma$ to a system having any of these values is at least 0.188562 .

This ad hoc study of this circuit leads to the conclusion that the obtained upper bound (0.186217) is useful to guarantee that, if we take any system (with physical meaning) at a distance from the system $\Sigma$ less than this upper bound, it will be controllable. That is to say, we can obtain any desired value for the voltage over the load $R$, with an appropriate source voltage $e_{S}(t)$ and an appropriate switching signal.

Example 2. Let us consider the following first order timevarying plant (see [18]) with the equations

$$
\dot{y}+2 y=0.1 u, \quad \dot{y}+0.25 y=10 u \text {. }
$$

Adopting a pole placement design, being the goal to place the poles of the closed-loop system at fixed locations, if we choose the state vector as $x=(\dot{u}, u, y)^{t}$, the following state space representation of the closed-loop system $\Sigma$ is obtained:

$$
\begin{aligned}
& \dot{x}(t)=A_{1} x(t)+B_{1} u_{c}(t), \\
& \dot{x}(t)=A_{2} x(t)+B_{2} u_{c}(t)
\end{aligned}
$$

with $u_{c}(t)=1$,

$$
\begin{array}{ccc}
A_{1}=\left(\begin{array}{ccc}
-4.4 & 1.56 & -43.2 \\
1 & 0 & 0 \\
0 & 0.1 & -2
\end{array}\right), & B_{1}=\left(\begin{array}{c}
12 \\
0 \\
0
\end{array}\right), \\
A_{2}=\left(\begin{array}{ccc}
-6.15 & -5.7 & 0.0225 \\
1 & 0 & 0 \\
0 & 10 & -0.25
\end{array}\right), & B_{2}=\left(\begin{array}{c}
0.12 \\
0 \\
0
\end{array}\right) .
\end{array}
$$

In this case,

$$
\begin{aligned}
& G_{1}(\Sigma)=\left(\begin{array}{llllllllllll}
B_{1} & A_{1} B_{1} & A_{1}^{2} B_{1} & A_{2} B_{1} & A_{1} A_{2} B_{1} & A_{1}^{2} A_{2} B_{1} & B_{2} & A_{1} B_{2} & A_{1}^{2} B_{2} & A_{2} B_{2} & A_{1} A_{2} B_{2} & A_{1}^{2} A_{2} B_{2}
\end{array}\right) \\
& =\left(\begin{array}{cccccccccccc}
12 & -52.8 & 251.0 & -73.8 & 343.4 & -1678.1 & 0.1 & -0.5 & 2.5 & -0.7 & 3.4 & -16.8 \\
0 & 12 & -52.8 & 12 & -73.8 & 343.4 & 0 & 0.1 & -0.5 & 0.1 & -0.7 & 3.4 \\
0 & 0 & 1.2 & 0 & 1.2 & -9.8 & 0 & 0 & 0.0 & 0 & 0.0 & -0.1
\end{array}\right) \text {, } \\
& G_{2}(\Sigma)=\left(\begin{array}{llllllllllll}
B_{1} & A_{2} B_{1} & A_{2}^{2} B_{1} & A_{1} B_{1} & A_{2} A_{1} B_{1} & A_{2}^{2} A_{1} B_{1} & B_{2} & A_{2} B_{2} & A_{2}^{2} B_{2} & A_{1} B_{2} & A_{2} A_{1} B_{2} & A_{2}^{2} A_{1} B_{2}
\end{array}\right) \\
& =\left(\begin{array}{cccccccccccc}
12 & -73.8 & 385.5 & -52.8 & 256.3 & -1272.7 & 0.1 & -0.7 & 3.9 & -0.5 & 2.6 & -12.7 \\
0 & 12 & -73.8 & 12 & -52.8 & 256.3 & 0 & 0.1 & -0.7 & 0.1 & -0.5 & 2.6 \\
0 & 0 & 120 & 0 & 120 & -558 & 0 & 0 & 1.2 & 0 & 1.2 & -5.6
\end{array}\right) .
\end{aligned}
$$


Since $\operatorname{rk} G(\Sigma)=\operatorname{rk}\left(G_{1}(\Sigma) \mid G_{2}(\Sigma)\right)=3$, system $\Sigma$ is controllable. In this case, $\operatorname{rk}_{1}(\Sigma) \mathrm{rk} G_{2}(\Sigma)=3$. Applying Theorem 3 , an upper bound for $\mu_{\mathbb{R}}\left(\left\{\left(A_{i}, B_{i}\right)_{i \in\{1,2\}}\right\}\right)$ with regard to the 2-norm is obtained:

$$
\sum_{i \in\{1,2\}} \sigma_{3}\left(1+\frac{\left\|A_{i}^{C}\right\|_{2}}{\sigma_{3, i}}\right)=123.756921,
$$

and applying Theorem 9 , an upper bound for $\mu_{\mathbb{R}}\left(\left\{\left(A_{i}\right.\right.\right.$, $\left.\left.B_{i}\right)_{i \in\{1,2\}}\right\}$ ) with regard to the 2-norm is obtained:

$$
\min _{\lambda_{1}, \lambda_{2} \in \mathbb{R}} \sigma_{3}\left(M\left(\Sigma, \lambda_{1}, \lambda_{2}\right)\right)=1.359749 \text {. }
$$

Thus, a substantial improvement for the upper bound has been achieved.

The ad hoc study of the closed-loop system $\Sigma$ leads to the conclusion that the obtained upper bound (1.359749) is useful to guarantee that, if we take any system (with physical meaning) at a distance from the system $\Sigma$ less than this upper bound, it will be controllable.

\section{Conclusions}

The main contribution of the paper is to obtain (Theorem 9) an upper bound for the distance from a controllable switched linear system to the set of uncontrollable ones, generalizing the upper bound obtained by D. Boley and W. S. Lu for a standard system. Up to now no bound for a switched linear system has been obtained before that in [16] and the one presented here, which improves it. On the other hand, this upper bound can be effectively computed with the current resources.

\section{Appendix}

\section{Eckart-Young-Mirsky Matrix Approximation Theorem}

Let the singular value decomposition (SVD) of $C \in \mathbb{R}^{m \times n}$ be given by $C=\sum_{i=1}^{r} \sigma_{i} u_{i} v_{i}^{t}$ with $r=\operatorname{rk} C$. If $k<r$ and $C_{k}=$ $\sum_{i=1}^{k} \sigma_{i} u_{i} v_{i}^{t}$, then

$$
\begin{gathered}
\min _{\operatorname{rk} D=k}\|C-D\|_{2}=\left\|C-C_{k}\right\|_{2}=\sigma_{k+1}, \\
\min _{\operatorname{rk} D=k}\|C-D\|_{F}=\left\|C-C_{k}\right\|_{F}=\sqrt{\sum_{i=k+1}^{q} \sigma_{i}^{2},}
\end{gathered}
$$

where $q=\min \{m, n\}$.

This theorem can be found in [19].

\section{Acknowledgment}

This work was supported by MTM 2011-23892.

\section{References}

[1] D. Boley, "Estimating the sensitivity of the algebraic structure of pencils with simple eigenvalue estimates," SIAM Journal on
Matrix Analysis and Applications, vol. 11, no. 4, pp. 632-643, 1990.

[2] J. Clotet, M. I. García-Planas, and M. D. Magret, "Estimating distances from quadruples satisfying stability properties to quadruples not satisfying them," Linear Algebra and Its Applications, vol. 332-334, pp. 541-567, 2001.

[3] D. L. Boley and W. S. Lu, "Measuring how far a controllable system is from an uncontrollable one," IEEE Transactions on Automatic Control, vol. AC-31, no. 3, pp. 249-251, 1986.

[4] R. Eising, "Between controllable and uncontrollable," Systems and Control Letters, vol. 4, no. 5, pp. 263-264, 1984.

[5] R. Eising, "The distance between a system and the set of uncontrollable systems," Memorandum COSOR 82-19, Eindhoven University of Technology, Eindhoven, The Netherlands, 1982.

[6] L. Elsner and C. He, "An algorithm for computing the distance to uncontrollability," Systems and Control Letters, vol. 17, no. 6, pp. 453-464, 1991.

[7] Zou Yun and C. Yang, "Formulae for the distance between controllable and uncontrollable linear systems," Systems and Control Letters, vol. 21, no. 2, pp. 173-180, 1993.

[8] Y. Ebihara and T. Hagiwara, "Computing the distance to uncontrollability via LMIs: lower and upper bounds computation and exactness verification," in Proceedings of the 45th IEEE Conference on Decision and Control (CDC '06), pp. 5772-5777, December 2006.

[9] L. Wu and W. X. Zheng, "Weighted $\mathscr{H}_{\infty}$ model reduction for linear switched systems with time-varying delay," Automatica, vol. 45, no. 1, pp. 186-193, 2009.

[10] L. Wu, W. X. Zheng, and H. Gao, "Dissipativity-based sliding mode control of switched stochastic systems," IEEE Transactions on Automatic Control, vol. 58, no. 3, pp. 785-791, 2013.

[11] G. Zhang, C. Han, Y. Guan, and L. Wu, "Exponential stability analysis and stabilization of discrete-time nonlinear switched systems with time delays," International Journal of Innovative Computing Information and Control, vol. 8, no. 3, pp. 1973-1986, 2012.

[12] L. Wu, X. Su, P. Shi, and J. Qiu, "A new approach to stability analysis and stabilization of discrete-time T-S fuzzy timevarying delay systems," IEEE Transactions on Systems, Man, and Cybernetics B, vol. 41, no. 1, pp. 273-286, 2011.

[13] C. H. Sun, Y. T. Wang, and C. C. Chang, "Switching T-S fuzzy model-based guaranteed cost control for two-wheeled mobile robots," International Journal of Innovative Computing Information and Control, vol. 8, no. 5, pp. 3015-3028, 2012.

[14] X. Su, P. Shi, L. Wu, and Y. D. Song, "A novel control design on discrete-time Takagi-Sugeno fuzzy systems with time-varying delays," IEEE Transactions on Fuzzy Systems, 2012.

[15] Z. Sun and S. S. Ge, Switched Linear Systems, Springer, London, UK, 2005.

[16] J. Clotet, J. Ferrer, and M. D. Magret, "Distance from a controllable switched linear system to an uncontrollable one," in Proceedings of the 2nd Meeting on Linear Algebra Matrix Analysis and Applications, pp. 1-7, Servicio de publicaciones de la UPV, Valencia, Spain, 2010.

[17] W. L. de Koning, "Digital optimal reduced-order control of pulse-width-modulated switched linear systems," Automatica, vol. 39, no. 11, pp. 1997-2003, 2003.

[18] D. J. Leith, R. N. Shorten, W. E. Leithead, O. Mason, and P. Curran, "Issues in the design of switched linear control systems: a benchmark study," International Journal of Adaptive Control and Signal Processing, vol. 17, no. 2, pp. 103-118, 2003. 
[19] S. van Huffel and J. Vandewalle, The Total Least Squares Problem: Computational Aspects and Analysis, vol. 9 of Frontiers in Applied Mathematics, SIAM, Philadelphia, Pa, USA, 1991. 


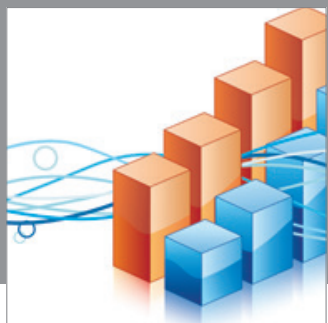

Advances in

Operations Research

mansans

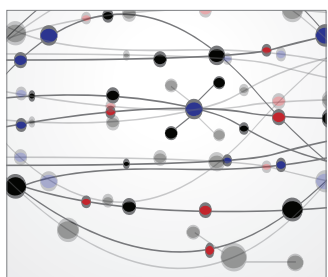

The Scientific World Journal
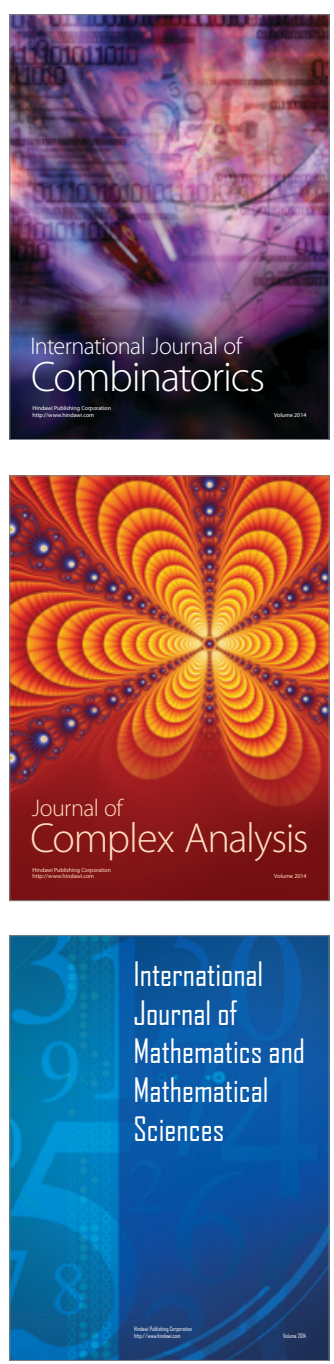
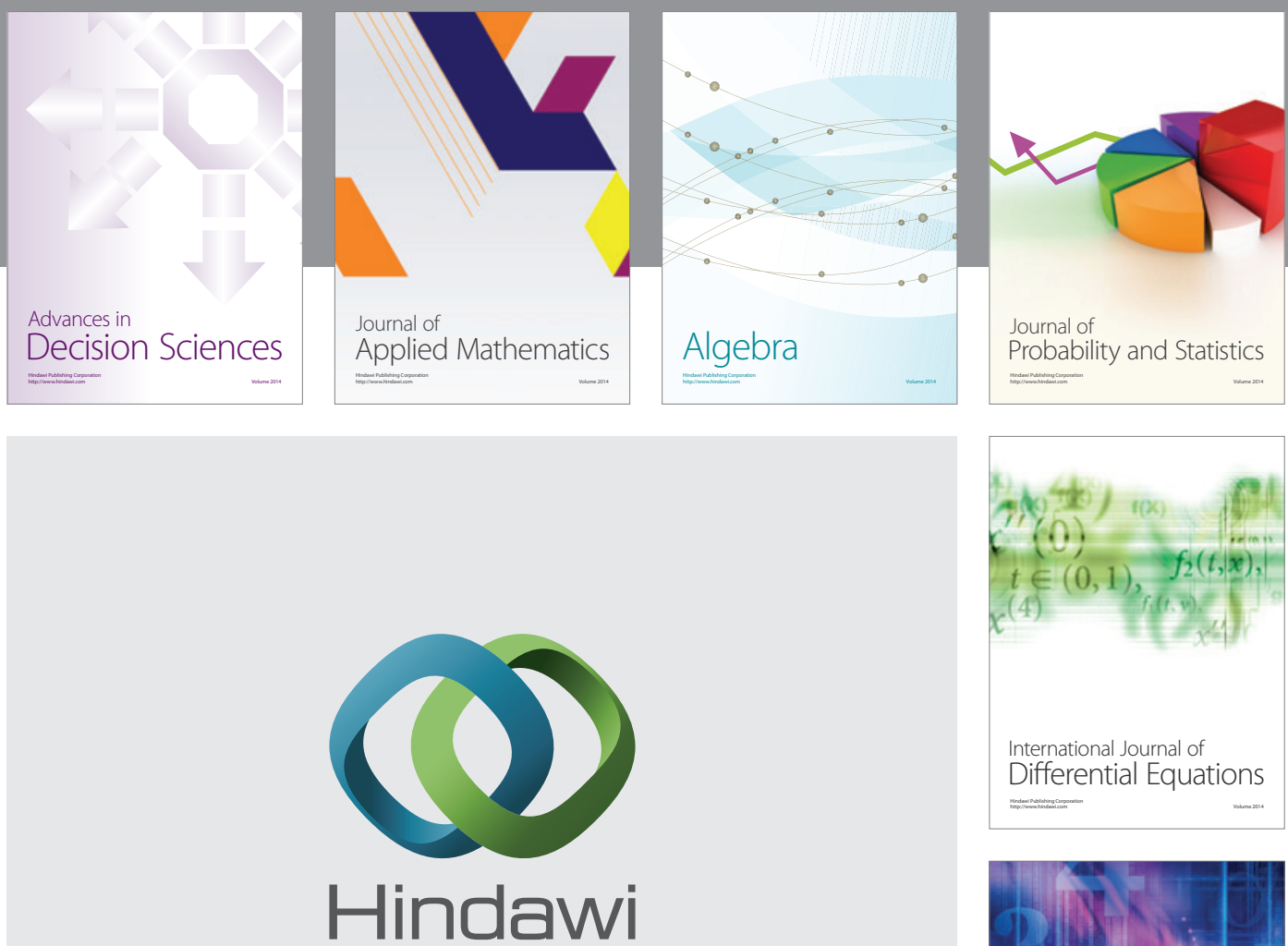

Submit your manuscripts at http://www.hindawi.com
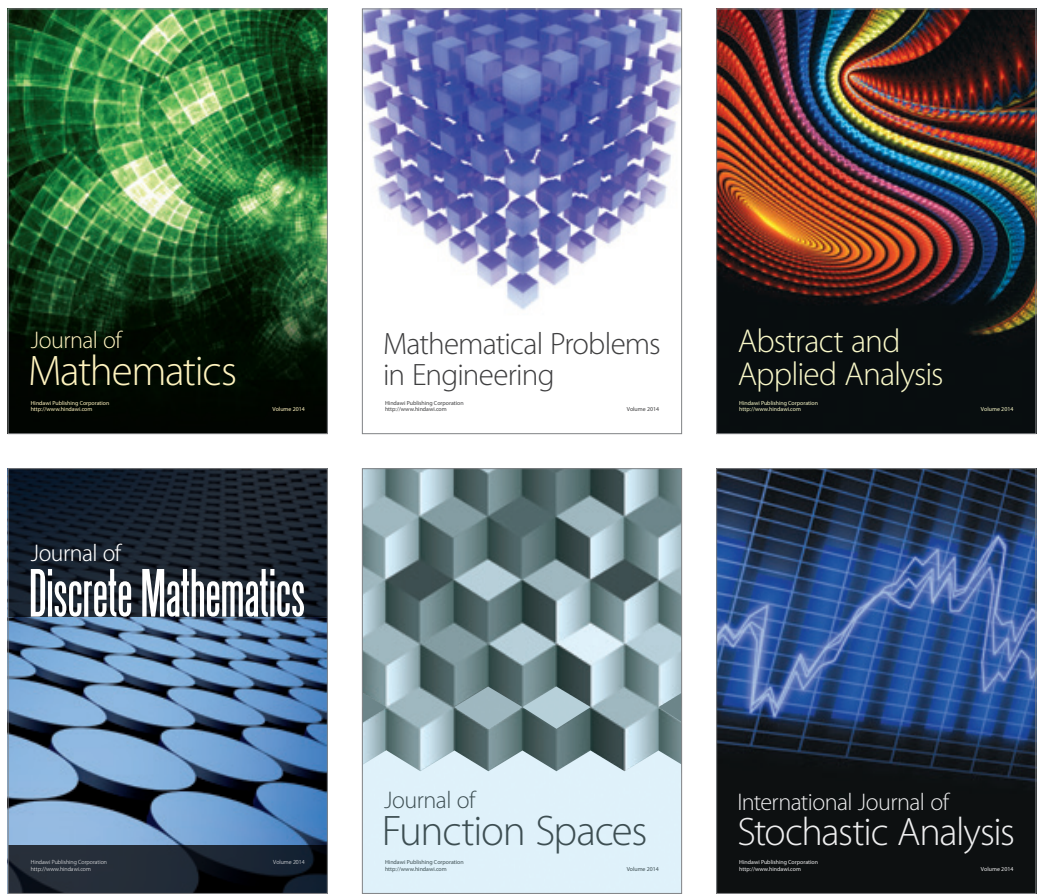

Journal of

Function Spaces

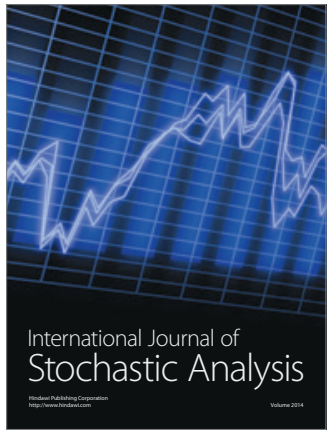

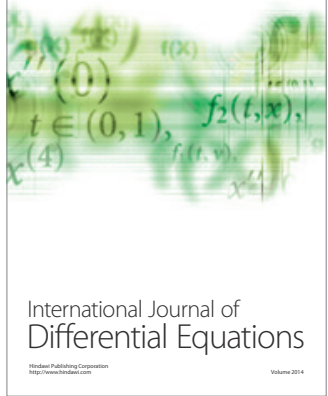
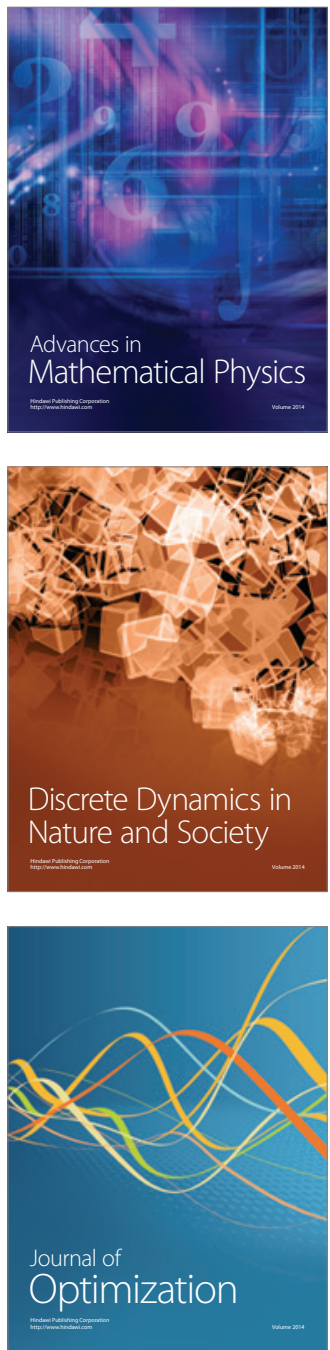